\title{
FOX01 Inhibits Tumor Cell Migration via Regulating Cell Surface Morphology in Non-Small Cell Lung Cancer Cells
}

\author{
Zhuo Gao ${ }^{a}$ Ruiqi Liub $\mathrm{Na} \mathrm{Ye}^{\mathrm{d}}$ Chao Liuc Xiuli Lie Xiaodong Guo ${ }^{\mathrm{a}}$ \\ Zhuoran Zhang $^{f}$ Xiaoxi Lig Yuanfei Yaoc Xiaofeng Jianga
}

aDepartment of Clinical Laboratory, the Fourth Affiliated Hospital of Harbin Medical University, Harbin, ' $F u$ dan University, Department of Oncology, Zhongshan Hospital, Shanghai, 'Department of Gastroenterology, the Third Affiliated Hospital of Harbin Medical University, Harbin, dDepartment of Obstetrics and Gynecology, Shijingshan Hospital, Beijing, eDepartment of Blood Transfusion, the First Affiliated Hospital of Harbin Medical University, Harbin, fDepartment of Pharmacy, the Fourth Affiliated Hospital of Harbin Medical University, Harbin, 9The Center of Metabolic Disease esearch, Nanjing Medical University, Nanjing, China

\section{Key Words}

Foxo1 • Cell migration • Cell surface morphology $•$ EMT $•$ NSCLC

\begin{abstract}
Background/Aims: Cell surface morphology plays pivotal roles in malignant progression and epithelial-mesenchymal transition (EMT). Previous research demonstrated that microvilli play a key role in cell migration of non-small cell lung cancer (NSCLC). In this study, we report that Forkhead box class O1 (FOXO1) is downregulated in human NSCLC and that silencing of FOXO1 is associated with the invasive stage of tumor progression. Methods: The cell proliferation, migration, and invasion were characterized in vitro, and we tested the expression of the Epithelial-mesenchymal transition (EMT) marker by immunofluorescence staining and also identified the effect of FOXO1 on the microvilli by scanning electron microscopy (SEM). Results: Functional analyses revealed that silencing of FOXO1 resulted in an increase in NSCLC cell proliferation, migration, and invasion; whereas overexpression of FOXO1 significantly inhibited the migration and invasive capability of NSCLC cells in vitro. Furthermore, cell morphology imaging showed that FOXO1 maintained the characteristics of epithelial cells. Immunofluorescence staining and western blotting showed that the E-cadherin level was elevated and Vimentin was reduced by FOXO1 overexpression. Conversely, the E-cadherin level was reduced and Vimentin was elevated in cells silenced for FOXO1. Furthermore, scanning electron microscopy (SEM) showed that FOXO1 overexpression increased the length of the microvilli on the cell surface, whereas FOXO1 silencing significantly reduced their length. Conclusions: FOXO1 is involved in human lung carcinogenesis and may serve as a potential therapeutic target in the migration of human lung cancer.

Z. Gao and R. Liu contributed equally to this work.

Xiaofeng Jiang

and Yuanfei Yao

Department of Clinical Laboratory, the Fourth Affiliated Hospital of Harbin Medical University Harbin (China)

E-Mail jiangxiaofeng@hrbmu.edu.cn, yaoyuanfei@hrbmu.edu.cn
\end{abstract}




\section{Cellular Physiology Cell Physiol Biochem 2018;48:138-148 \begin{tabular}{l|l} 
DOI: 10.1159/000491670 & $\begin{array}{l}\text { O } 2018 \text { The Author(s). Published by S. Karger AG, Basel } \\
\text { www.karger.com/cpb }\end{array}$
\end{tabular}}

Gao et al.: FOXO1 Inhibits Cell Migration by Regulating the Length of Microvilli

\section{Introduction}

Lung cancers can be classified into two major types according to their pathological characteristics: Small cell lung cancer (SCLC) and non-small cell lung cancer (NSCLC). The latter can be further divided into a variety of cancer subtypes according to their heterogeneous morphology and cell origin, which are named for the type of cell found in the tumor, such as adenocarcinoma, squamous cell carcinoma, and large cell carcinoma [1, $2]$. These subtypes of NSCLC account for $\sim 85 \%$ of all lung cancer cases $[3,4]$. The mortality rate of NSCLC in many countries is increasing because of its high metastatic potential $[5,6]$. Cancer metastasis is closely related to cell migration and motility; therefore, it is important to identify the underlying mechanisms of motility and migration of NSCLC.

Studies have linked the tumor suppressor activity of Forkhead box transcription factor 0 proteins (FOXOs) to the regulation of genes involved in angiogenesis, apoptosis, cell cycle arrest, cell metabolism, oxidative stress, immune regulation, differentiation, and cell death [7-11]. In multiple cancer types, FOXO3 overexpression reduces cell viability and invasiveness [12]. FOXO3 downregulation increases the expression of TWIST1 and cell motility $[13,14]$. Similarly, FOXO4 downregulation by the PI3K-AKT pathway correlates with metastasis. In addition, it limits prostate cancer cell migration and invasion in vitro and in vivo [15]. Moreover, FOXO1 has been shown to negatively regulate RUNX2 transcriptional activity, RUNX2-mediated migration, and the invasion of prostate cancer cells. A recent study suggested that FOXO1 is also involved in the promotion of breast tumor cell invasion [16]. However, the mechanisms of FOXO1-mediated cell motivations in NSCLC remain poorly understood.

Epithelial-mesenchymal transition (EMT) is a developmental mechanism of tumor progression that is frequently observed in various types of cancers. Cancer cells that undergo EMT have increased metastatic properties. Increasing evidence shows that EMT is related to a high mortality rate and poor prognosis $[17,18]$. During EMT, epithelial cells reorganize their cytoskeleton to alter cell polarity and morphology, and lose their junctions, which increases the invasive capacity of the cells. The hallmarks of EMT include decreased expression of E-cadherin and overexpression of Vimentin [19, 20]. In recent years, it was observed that EMT is closely related to carcinoma progression, and acts as a major driver of cellular morphogenesis and tumor progression [21]. Although EMT is able to modulate cell surface ultrastructure, its regulatory mechanism of the changes in cellular ultrastructure in tumor metastasis is largely unknown.

In the current study, we knocked down FOXO1 in the NSCLC cell line H1792 using RNA interference (RNAi) and overexpressed FOXO1 in the NSCLC cell line H520 using a recombinant plasmid. We analyzed the biological behavior of the cells, including the expression and cellular location of epithelial and mesenchymal markers, such as E-cadherin and Vimentin, and the changes of the cell ultrastructure in NSCLC. Our results demonstrated that overexpression of FOX01 suppressed NSCLC proliferation and invasion in vitro. Furthermore, FOXO1 inhibited the EMT-like phenotype by increasing the length of the microvilli on the cell surface. These findings suggested that FOXO1 is a potential tumor suppressor gene in NSCLC development and progression, as well as a therapeutic target in NSCLC metastasis.

\section{Materials and Methods}

\section{Cell culture}

Human bronchial epithelial cell line 16HBE, and human NSCLC cell lines H1792, H520, and H1650, H460 were purchased from the American Type Culture Collection (ATCC, Manassas, VA, USA). These cells were identified by the cell banks using short tandem repeat analysis. 16HBE cells were isolated from the normal human bronchial epithelium. H520 is a cell line from squamous cell carcinoma of the lung, H1792 is a cell line from adenocarcinoma cell carcinoma of the lung, H1650 is cell line from metastatic lung and 


\section{Cellular Physiology Cell Physiol Biochem 2018;48:138-148 \begin{tabular}{l|l} 
DOI: 10.1159/000491670 & $\begin{array}{l}\text { O } 2018 \text { The Author(s). Published by S. Karger AG, Basel } \\
\text { www.karger.com/cpb }\end{array}$
\end{tabular} \\ Gao et al.: FOXO1 Inhibits Cell Migration by Regulating the Length of Microvilli}

pleural effusion and H460 is a cell line from large cell carcinoma of the lung. Stable cell line H520/FOXO1 was maintained in our laboratory. Cells were cultured in Roswell Park Memorial Institute (RPMI 1640) medium (Invitrogen, Carlsbad, CA, USA). All media were supplemented with 10\% fetal bovine serum (Invitrogen), and cell lines were maintained at $37^{\circ} \mathrm{C}$ in a humidified atmosphere containing $5 \% \mathrm{CO}_{2}$.

\section{Clinical samples}

Thirty paired lung cancer and benign adjacent non-cancerous tissue samples were analyzed using real-time quantitative reverse transcription PCR (qRT-PCR). This study was approved by the Institutional Review Board of the Third Affiliated Hospital of Harbin Medical University, and written informed consent was obtained from all patients.

\section{Immunohistochemical staining}

Immunohistochemical staining was determined by the staining intensity and the percentage of immunoreactive cells. 6- $\mu \mathrm{m}$-thick sections were prepared by cutting paraffin-embedded colon cancer tissues. After deparaffinization and rehydration, $3 \mathrm{H}_{2} \mathrm{O}_{2}$ was used to block endogenous peroxidase activity. Furthermore, microwave treatment was used to retrieve the antigen before blocking nonspecific reactions using $5 \%$ bovine serum albumin. The sections were then incubated with primary antibodies against FOXO1 (Abcam, Cambridge, MA, USA) at $4{ }^{\circ} \mathrm{C}$ overnight. After incubation with streptavidin-biotin peroxidaselabeled secondary antibody for $1 \mathrm{~h}$ at room temperature, 3, 3'-diaminobenzidine (DAB) substrate (ZSGB Bio, Beijing, China) was applied for staining according to the manufacturer's instructions. Negative control staining was performed by replacing the primary antibodies with phosphate-buffered saline (PBS). The results were evaluated by a pathologist who was blinded to the clinical information. The staining intensity was scored as follows: 0 (negative), absent or staining $<5 \%$ of cells; grade 1 , mild to moderate staining of $5-50 \%$ of cells; grade 2 , moderate to intense staining of more than $50 \%$ of the cells.

\section{Scanning Electron Microscopy (SEM) Analysis}

The cells were cultured in RPMI 1640 for $24 \mathrm{~h}$, washed with PBS three times, and placed in 4\% paraformaldehyde (PFA) for $1 \mathrm{~h}$ at $37^{\circ} \mathrm{C}$. The cells were then washed with PBS five times and treated with $1 \%$ osmium tetroxide (Sigma-Aldrich, St. Louis, MO, USA) for $1 \mathrm{~h}$ in PBS at room temperature. Dehydration was then accomplished by submersing the samples in an ethanol gradient $(20 \%, 50 \%, 75 \%, 90 \%$, and $100 \%$ ) for 5 minutes at room temperature. The samples were then coated with a thin film of gold using a sputter Q150TES at $20 \mathrm{~mA}$ for 10 seconds before viewing by SEM (Quanta FEG 250 (FEI)) at $3 \mathrm{KV}$.

\section{Transfection and Western Blotting}

For transient transfection, $4 \times 10^{5}$ cells were plated into 6-well plates and kept in antibiotic-free medium for $12 \mathrm{~h}$ before transfection. The cells were then transfected with the short interfering RNA (siRNA) or plasmid using Lipofectamine 2000 (Invitrogen) according to the manufacturer's instructions. The FOXO1 siRNA sequence was 5'- CGGAGAAUGUAUACAAGCATT-3'. For western blotting, cells and specimens were washed with ice cold PBS and lysed in Radioimmunoprecipitation assay (RIPA) buffer with $0.1 \%$ protease inhibitor cocktail III (Calbiochem, San Diego, CA, USA). Proteins $(25 \mu \mathrm{g})$ were loaded onto a 12\% SDSPAGE gel, electrophoresed, and then transferred to polyvinylidene fluoride (PVDF) membranes (Millipore, Bedford, MA, USA). After blocking with 5\% skimmed milk, the membranes were incubated with anti-FOXO1 (1:1000 dilutions, Abcam, CA, USA), anti-GAPDH (1:5000 dilutions, Santa Cruz Biotechnology, Santa Cruz, CA, USA) at $4{ }^{\circ} \mathrm{C}$ overnight, followed by incubating with horseradish peroxidase conjugated-secondary antibody (ZSGB-BIO, Beijing, China) for $1 \mathrm{~h}$ at room temperature, and the immunoreactive bands were visualized using ECL detection reagents (Millipore). GAPDH served as the loading control.

\section{qRT-PCR}

Total RNA was extracted using the Trizol reagent (Invitrogen) according to the manufacturer's instructions. Total RNA (1 $\mu \mathrm{g})$ was converted to first-strand cDNA using SuperScript II Reverse Transcriptase (Invitrogen). The qRT-PCR analysis was performed with appropriate primers on a ViiA 7 Real-Time PCR System (Applied Biosystems, Foster City, CA, USA), using the Fast SYBR Green Master Mix System (Invitrogen) according to the manufacturer's instructions. The primer sequences were: FOXO1 sense 5'-CAGCAAATCAAGTTATGGAGGA-3' and antisense 5'-CTGAGAGGAGGGGTGTTACTAT-3'; E-cadherin 


\section{Cellular Physiology Cell Physiol Biochem 2018;48:138-148

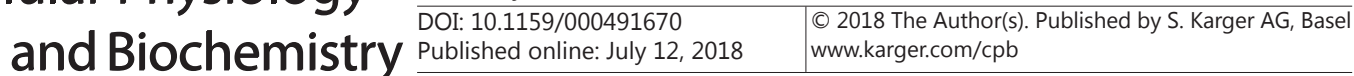

Gao et al.: FOXO1 Inhibits Cell Migration by Regulating the Length of Microvilli

sense 5'-GTCGAGGGAAAAATAGGCTG-3' and antisense 5'-GCCGAGAGCTACACGTTCAC-3'; Vimentin sense 5'-GACAGAGTGTCACTCTGTTGCC-3' and antisense 5'- ATGCCTGTAGTCCCAGCTAC-3'; GAPDH sense 5'-AACAGCCTCAAGATC ATCAGC-3' and antisense 5'-GGATGATGTTCTGGAGAGCC-3'. The level of GAPDH was quantified as a control.

\section{Cell Proliferation Assay}

3-(4, 5-dimethylthiazol-2-yl)-2, 5-diphenyltetrazolium bromide (MTT) assays were used to evaluate the ability of cell proliferation. For the MTT assay, $24 \mathrm{~h}$ after transfection, $3 \times 10^{3}$ cells per well were seeded in 96-well plates, each with eight replicas. The cells were then incubated with $0.5 \mathrm{mg} / \mathrm{ml}$ MTT (SigmaAldrich) at $37^{\circ} \mathrm{C}$ for $4 \mathrm{~h}$. The medium was removed, and the precipitated Formosan was dissolved in $150 \mu \mathrm{l}$ dimethyl sulfoxide (DMSO). The absorbance of the solution was measured at $490 \mathrm{~nm}$ using a micro-plate auto-reader (Bio-Rad, Richmond, CA, USA). The cells were treated and measured every day for 5 days.

\section{Transwell Invasion Assay}

A matrigel invasion assay was performed using Millipore Trans-well chambers ( $8 \mu \mathrm{m}$ : pore size); 2 $\times 10^{4}$ FOX01-transfected AH520 cells or siFOX01-transfected NCI-H1792 cells were seeded in the upper chamber of a 12-well plate, which was coated with Matrigel (Corning), in $500 \mu \mathrm{l}$ of RPMI 1640 medium without serum. The lower chamber was filled with $500 \mu$ l RPMI 1640 medium with 15\% FBS to induce cell migration. The chamber was incubated at $37^{\circ} \mathrm{C}$, for $24 \mathrm{~h}$. At the end of incubation, cells in the upper surface of the membrane were removed with a cotton swab. Migrated cells on the lower surface of the membrane were stained with Giemsa (HiMedia). The images were obtained using a CKX41 inverted microscope (Olympus) and the cells were counted in eight different view fields, using ImageJ software. The experiment was conducted in triplicate.

\section{Transwell Migration Assay}

Transwell migration was performed using a culture plate with $8.0 \mathrm{~mm}$ polyethylene terephthalate (PET) insert-strips (BRAND, Wertheim, Germany). Cells $\left(3 \times 10^{4}\right)$ cells were plated into the top chamber in $0.2 \mathrm{ml}$ of RPMI-1640 medium without FBS. The lower chambers were placed into a culture plate, each well containing $600 \mathrm{ml}$ of RPMI-1640 medium with $10 \%$ fetal calf serum to act as the nutritional attractant. After incubation for $12 \mathrm{~h}$ at $37{ }^{\circ} \mathrm{C}$, the cells that had traversed the membrane were fixed with $20 \%$ methanol and stained by crystal violet for $15 \mathrm{~min}$. The cells from three different fields in each chamber were counted and expressed as the average number of cells per field of view using a phase contrast microscope.

\section{Cell Scratch Wound Healing Assay}

For the scratch wound healing assay, FOX01-transfected AH520 cells, siFOXO1-transfected NCI-H1792 cells, and control cells were directly seeded on 12 well plates after trypsinization. Sixteen hours later, a 3 $\mathrm{mm}$ wound was introduced across the diameter of each plate, and the suspended cells were washed away with PBS three times. The cells were then cultured in a medium with $1 \%$ FBS. Cell migration was observed by microscopy at $0 \mathrm{~h}$ and $16 \mathrm{~h}$. The wound width was analyzed objectively using Image J.

\section{Immunofluorescent Staining}

Cells seeded on glass coverslips in 12-well plates were fixed in $4 \%$ formaldehyde solution in PBS buffer (pH 7.4) for $15 \mathrm{~min}$ at room temperature, and then permeabilized with $0.1 \%$ Triton X-100 10 min at room temperature. After blocking in 3\% bovine serum albumin in PBS for $30 \mathrm{~min}$ at room temperature, specific antibodies were used to detected E-cadherin, and Vimentin. Following incubation with donkey antiRabbit IgG AlexaFluor-488 or donkey anti-mouse IgG AlexaFluor-568 (Invitrogen), the cells were stained with 2-(4-amidinophenyl)-1H-indole-6-carboxamidine (DAPI, Burlingame, CA, USA). Finally, images were captured under a Zeiss LSM 510 META confocal microscope.

\section{Cell Spreading Assay}

The assays were performed as described previously with the following modifications [22]. Briefly, cells at $80 \%$ confluence were detached using EDTA-trypsin, and RPMI 1640 with 10\% FBS was added to terminate trypsinization immediately. The FOX01-transfected H520 cells, siFOXO1-transfected H1792 cells, and control cells were then resuspended in RPMI 1640 containing 10\% FBS and seeded on coverslips in 


\section{Cellular Physiology Cell Physiol Biochem 2018;48:138-148

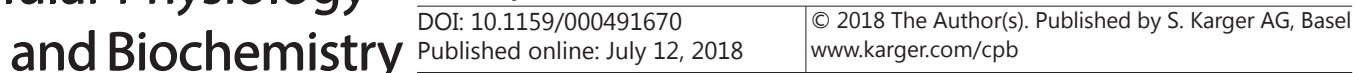 \\ Gao et al.: FOXO1 Inhibits Cell Migration by Regulating the Length of Microvilli}

12-well dishes and cultured. Cell spreading was monitored at different time points. Cells were then washed with PBS and fixed with $4 \%$ paraformaldehyde. Photographs were taken at $40 \times$ magnification under a phase contrast microscope (Leica DM IRB). The cell surface area ( $\mu \mathrm{m}^{2}, 25-50$ cells) was quantified using Axiovision Software ${ }^{\mathrm{TM}}$ Rel 4.7 (Zeiss).

\section{Statistical analysis}

All data are presented as the mean \pm standard deviation (SD), and mean values were compared by using Student's t-test. Results were considered statistically significant when $p<0.05$ was obtained. The statistical analyses were performed in Statistical Package for Social Science (SPSS; version 18.0) software.

\section{Results}

FOXO1 is significantly downregulated in NSCLC

To gain insights into the role of F0X01 in NSCLC, we analyzed the protein and mRNA expression levels of FOXO1 in lung cancer and benign adjacent non-cancerous tissues $(\mathrm{n}=30)$ by immunohistochemical staining and qRTPCR. The results of immunohistochemical staining suggested that FOX01 expression in cancer was lower (Fig. 1A and B). As shown in 1C, the expression of the FOXO1 mRNA was significantly downregulated in $80 \%(24 / 30)$ of the cases, compared with that in the corresponding adjacent normal lung tissues. FOX01 levels were consistently lower in most of the NSCLC cell lines, including H1650, H460, and H520 cell lines, compared with that in the human bronchial epithelial cell line 16HBE (Fig. 1D and E). To investigate the localization of the endogenous expression of FOX01 by immunofluorescence, the subcellular localization of FOXO1 was determined in H1792. We also detected the localization of FOXO1 in H520/ Fox01-GFP cells. The results showed that F0X01 is mainly localized in the nucleus (Fig. 1F). Furthermore, we detected the proliferation of these cell lines (Fig. 1G). Among the four NSCLC cell lines, the proliferation of H1792 was lower than the others, which contrasted with the F0X01 expression. The localization of F0X01 in the nucleus may suggest an important tumor suppressing function. In summary, our results provided the evidence of FOXO1 being a potential tumor suppressor gene associated with lung cancer progression.

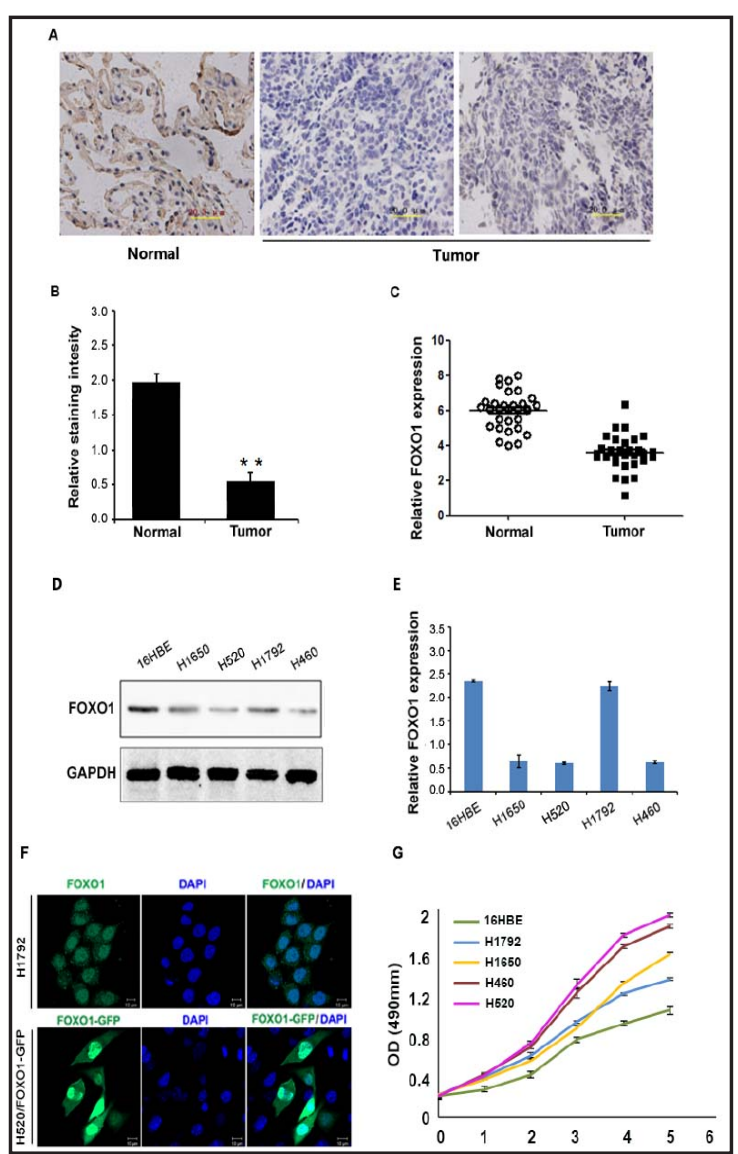

Fig. 1. FOXO1 expression in NSCLC tissues and cell lines. (A), (B) Immunohistochemical analysis showing FOXO1 levels in lung cancer tissues and adjacent lung tissues. Scale bar, $100 \mu \mathrm{m}$. (C) Real-time PCR analyses of FOX01 were performed in 30 paired human tumor and normal tissues. (D, E) Western blotting and realtime PCR analysis showing FOX01 level in various lung cancer cell lines. (F) Immunofluorescence staining to detect the localization of FOX01. Scale bar, $10 \mu \mathrm{m}$. (G) The proliferation of H1792, H1659, H520, H460, and H16BE cells. 
Fig. 2. FOXO1 regulates NSCLC cell proliferation. (A) The protein levels of FOXO1 in H520 and H460 transfected with FOXO1, or H1792 and H1650 cells transfected with FOX01-si, as determined by western blotting and qRT-PCR analysis. GAPDH was used as a loading control. (B) MTT cell proliferation assays in H520/FOXO1 and H460/FOX01; *p<0.05, ** $\mathrm{p}<0.001$ compared with control cells. (C) MTT cell proliferation assays in H1792/FOXO1si compared with scrambled siRNA transfected cells. and H1650/FOX01si cells; ${ }^{*} \mathrm{p}<0.05$, ${ }^{* *} \mathrm{p}<0.001$

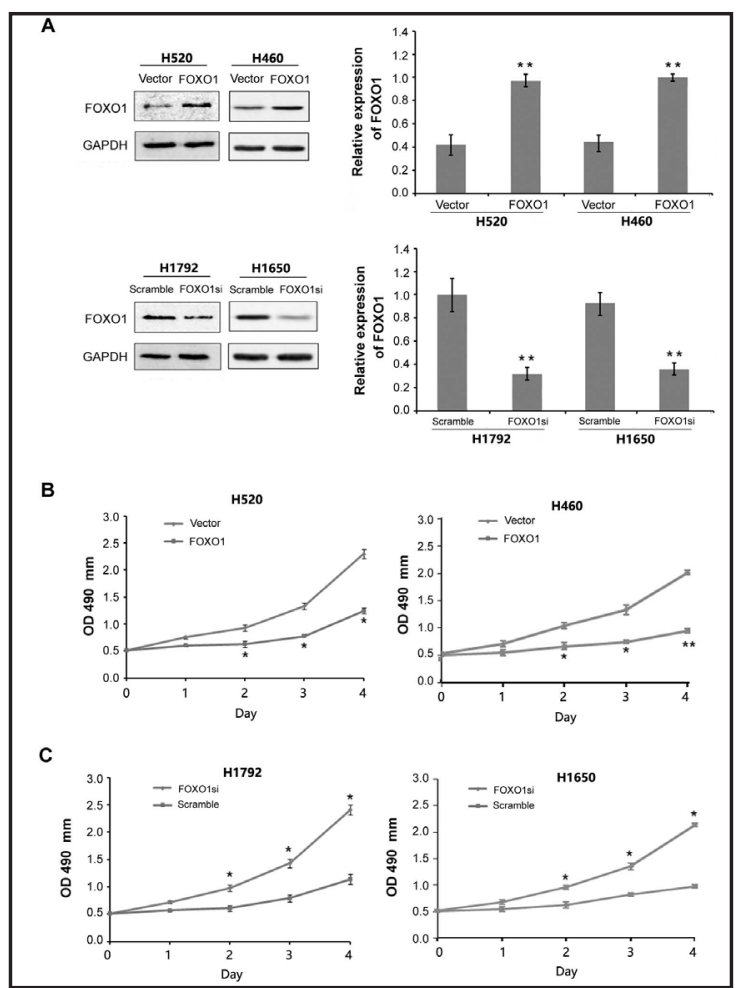

Fig. 3. FOXO1 inhibits the migration and invasion of NSCLC cells. (A) Cell migration and (B) cell invasion as determined by Transwell analysis in H520/FOXO1 and H1792/FOX01si cells compared with control or scramble cells; ${ }^{*} \mathrm{p}<0.05$, ${ }^{* *} \mathrm{p}<0.001$. (C) Wound-healing assay to assess the effect of FOXO on cell mobility in H520/FOXO1 and H1792/ F0X01si cells. The cells were measured over $16 \mathrm{~h}$ by wound-healing assay. Statistical analysis showed the percentage of cell migration. (D) Statistical analysis showing the percentage of cell migration.
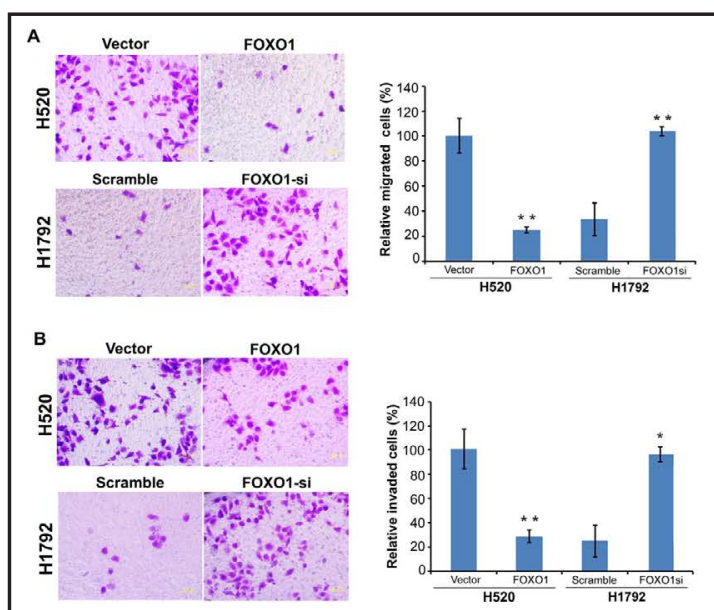

c
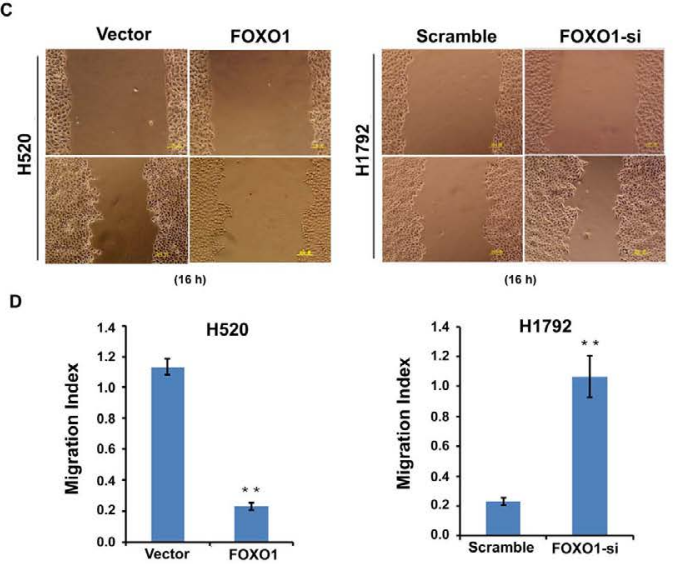


\section{Cellular Physiology Cell Physiol Biochem 2018;48:138-148

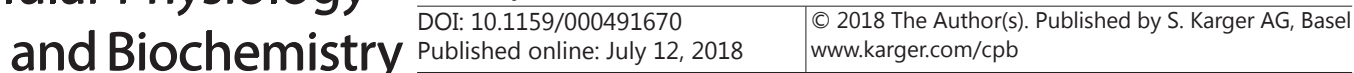 Gao et al.: FOXO1 Inhibits Cell Migration by Regulating the Length of Microvilli}

Overexpression of FOXO1 suppresses NSCLC cell proliferation in vitro

To investigate the role of FOXO1 in lung cancer progression, we overexpressed FOXO1 in H520 and H460 cells, and used siRNAs targeting FOXO1 to inhibit FOXO1 expression in $\mathrm{H} 1792$ and $\mathrm{H} 1650$ cells. The effective knockdown and overexpression of FOX01 was confirmed by qRT-PCR and western blotting. Compared with the control cells, FOX01-transfected cells showed high FOXO1 expression. FOXO1 siRNA-transfected cells showed significantly reduced FOX01 expression (Fig. 2A). We assessed the effect of FOXO1 alteration on NSCLC cell proliferation in FOX01 overexpressing cells and si-FOXO1 cells using the MTT assay. As shown in Fig. $2 \mathrm{~B}$ and $\mathrm{C}$, increased FOXO1 significantly inhibited H520/FOXO1 and H460/FOXO1 cell proliferation compared with the vector-only cells. In addition, reduced FOX01 expression significantly increased cell proliferation in H1792/siFOXO1 and H1650/siFOX01cells, compared with the scrambled siRNA cells.

FOXO1 inhibits cell invasion and migration of NSCLC cells

To determine the role of FOXO1 in cell invasion, Transwell migration assays were performed in H520/FOXO1 and H1792/FOX01si cells. We observed that $105 \pm 9$ H520/Vector cells invaded the Transwell membrane. In Fig. $3 \mathrm{~A}$ and $\mathrm{B}$, H520/FOXO1 decreased the average number of invading cells to $41 \pm 6$, whereas silencing of FOXO1 resulted in $107 \pm 8$ invading cells. There was significant suppression of cell invasion between FOX01 overexpressing and control cells $(p<0.05)$, and silencing FOX01 promoted invasion compared with the cells transfected with the scrambled siRNA $(p<0.05)$. Furthermore, to determine the role of FOXO1 in cell migration, a cell scratch healing assay was performed in H520/FOXO1 and H17921/FOX01si cells. Decreased cell migration was correlated with FOX01 overexpression. In contrast, the cell migration ability was significantly enhanced by si-FOX01. Thus, the role of FOXO1 in cell migration was examined in lung cancer cells. As shown in Fig. $3 \mathrm{C}$ and D, the migration of H520/FOXO1 cells was significantly lower compared with that in H520/Vector cells $(p<$ $0.001)$.

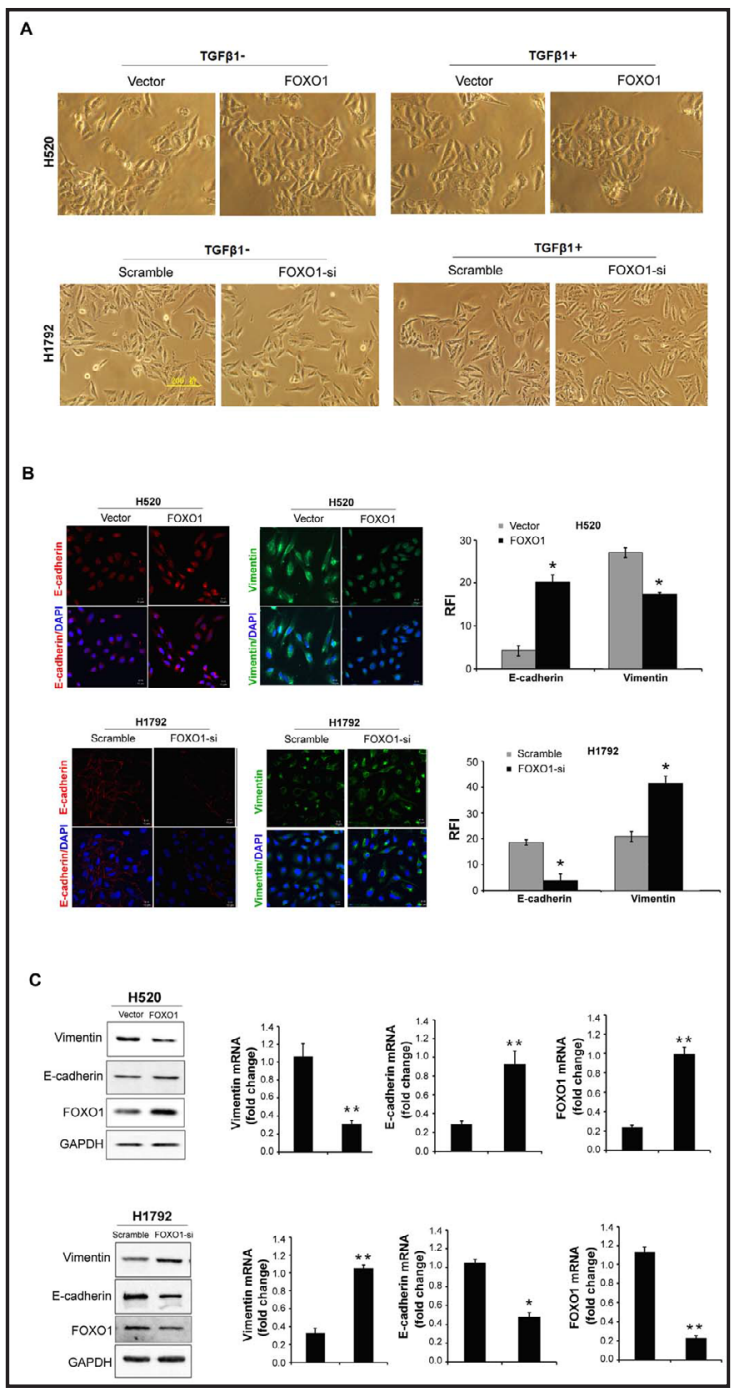

Fig. 4. FOXO1 inhibits TGF- $\beta 1$-induced EMT in NSCLC cells. (A) Morphology of H520/vector, H520/FOX01, H1792/siCtrl, and H1792/FOX01si cells with or without TGF- $\beta 1$ treatment (10 ng/ml) for $24 \mathrm{~h}$. (B) E-cadherin and Vimentin in H520/FOXO1 and H1792/ FOX01si cells with TGF- $\beta 1$ treatment $(10 \mathrm{ng} / \mathrm{ml})$ for $48 \mathrm{~h}$ were detected by immunofluorescence staining. Statistical analysis showed the relative fluorescence intensity of E-cadherin or Vimentin. Quantification of the fluorescent intensity was performed using Image J. Values represent means \pm SD of three independent experiments. * indicates $\mathrm{p}<0.05^{*}$. (C) E-cadherin and Vimentin in H520/FOX01 and H1792/FOXO1si cells after TGF- $\beta 1$ treatment $(10 \mathrm{ng} / \mathrm{ml})$ for $48 \mathrm{~h}$ were detected by western blotting and real-time PCR. 


\section{Cellular Physiology Cell Physiol Biochem 2018;48:138-148 \begin{tabular}{c|c|c|} 
DOI: 10.1159/000491670 & $\begin{array}{l}\text { O } 2018 \text { The Author(s). Published by S. Karger AG, Basel } \\
\text { www.karger.com/cpb }\end{array}$
\end{tabular} Gao et al.: FOXO1 Inhibits Cell Migration by Regulating the Length of Microvilli}

Silencing of FOXO1 is linked to the EMT-like phenotype

EMT is involved in tumor progression and an aggressive phenotype of lung cancer. EMT implicates loss of epithelial markers, and the concomitant acquisition of mesenchymal markers. To investigate the role of FOXO1 in EMT, H520/FOXO1, H1792/FOXO1-si, and control cells were stimulated with or without TGF- $\beta 1(10 \mathrm{ng} / \mathrm{ml})$ for $24 \mathrm{~h}$. As shown in Fig. 4A, H520/FOXO1 cells did not show an obvious EMT phenotype in response to TGF- $\beta 1$ stimulation, whereas H1792/FOX01si cells treated with TGF- $\beta 1$ acquired a more elongated and migratory morphology compared with H1792/scramble cells. This suggested that FOX01 has an inhibitory effect on EMT. Immunofluorescence staining showed that the E-cadherin levels were elevated in H520/FOXO cells; however, Vimentin levels were reduced. Furthermore, compared with H17921/scramble cells, FOX01 knockdown in H17921/ F0X01si cells led to less E-cadherin but more Vimentin expression in response to TGF- $\beta 1$ treatment for $48 \mathrm{~h}$ (Fig. 4B). Our results indicated that overexpression of FOXO1 in H520 cells led to a reduction of Vimentin expression and a restore expression of E-cadherin after TGF- $\beta 1$ treatment, as assessed by western blotting and q-RT-PCR assays (Fig. $4 \mathrm{C}$ ). Collectively, these results indicated that FOXO1 inhibits the migration of NSCLCs by suppressing EMT.

FOXO1 inhibits NSCLC malignancy by regulating the ultrastructure of the cell surface

The cell surface ultrastructure plays an important role in cell adhesion and migration. Aberrant regulation of the cell ultrastructure has been identified in various cancers. To investigate how FOXO1 regulates cell surface ultrastructure, SEM analysis was performed on H520/FOXO1 and H1792/FOX01si cells (Fig. 5A and C). As shown in Fig. 5B and D, overexpression of FOXO1 in H520/FOXO1 cells increased the length of the microvilli $(p<0.05)$, whereas FOX01 silencing significantly reduced the length of the microvilli $(p<$ $0.05)$. The SEM analysis suggested that the increased length of microvilli induced by FOX01 plays a key role in ultrastructural alteration. To gain further insights into cell spreading, we tested cell spreading at different time intervals after plating the H520/FOXO1, H1792/siFOXO1, and control cells. We found that approximately $84 \%$ of FOX01-overexpressing cells spread at $2 \mathrm{~h}$ after plating, whereas 58\% of control cells spread at $2 \mathrm{~h}$ after plating. In contrast, only about $56 \%$ of si-FOXO1 cells and $78 \%$ of the control cells spread at $2 \mathrm{~h}$ (Fig. 5E). The result suggested that FOXO1 promotes the cell spreading process significantly, whereas si-FOXO1 slows down cell spreading.

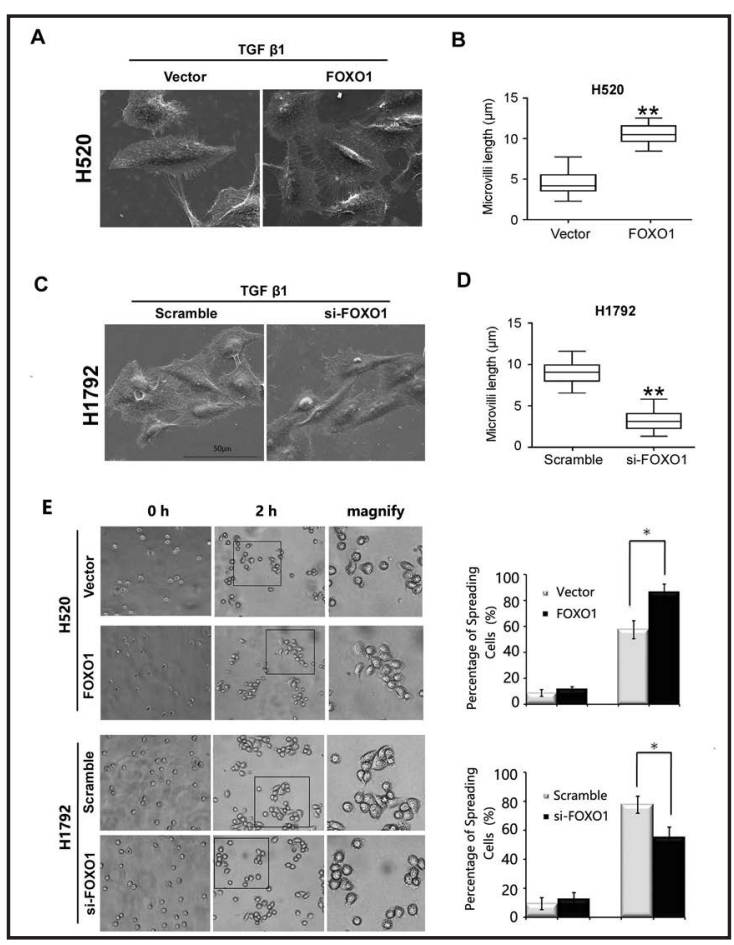

Fig. 5. FOXO1 alters cell surface ultrastructure. (A) Scanning election microscope images of H520/FOXO1 and H520/Vector cells with TGF- $\beta 1$ treatment (10 ng/ $\mathrm{ml}$ ) for $48 \mathrm{~h}$. (B) The box and whisker graph represent the length of the microvilli in H520/FOX01 and H520/ Vector cells, which were measured using the Line Tool of Image J software. (C) Scanning election microscope images of H1792/scramble and H1792/FOX01si cells with TGF- $\beta 1$ treatment ( $10 \mathrm{ng} / \mathrm{ml}$ ) for $48 \mathrm{~h}$. (D) The box and whisker graph represent the length of the microvilli in H1792/scramble and H1792/FOXO1si cells. (E) H520/FOX01, H1792/siFOX01 and control cells spread on the surface of 6-wells plate at the indicated time points: 0 and $2 \mathrm{~h}$. Scale bar, $100 \mathrm{~mm}$. 


\section{Cellular Physiology Cell Physiol Biochem 2018;48:138-148 \begin{tabular}{l|l} 
DOI: 10.1159/000491670 & $\begin{array}{l}\text { O } 2018 \text { The Author(s). Published by S. Karger AG, Basel } \\
\text { www.karger.com/cpb }\end{array}$
\end{tabular}}

Gao et al.: FOXO1 Inhibits Cell Migration by Regulating the Length of Microvilli

\section{Discussion}

FOXO is one of the most studied subgroups of forkhead transcription factors. This family consists of four members FOXO1, FOXO3, FOXO4, and FOXO6. The activity of FOXO transcription factors is mainly regulated by covalent modifications. Forkhead box protein 01 (FOX01) is a major target protein for activated P13K/AKT signaling [23, 24]. FOXO1 orchestrates programs of gene expression that regulate cell proliferation, apoptosis, and transition. FOXO1 proteins are regulated by phosphorylation, ubiquitination, and acetylation via several kinases [25]. By contrast, AMPPK and JNK were suggested to induce nuclear translocation and activation of FOXO1 proteins [26]. In addition, FOXO transcription factors regulate different cellular processes, such as inhibition of proliferation, induction of differentiation or apoptosis, and protection against oxidative damage. Several recent studies have shown that there is an association between FOXO1 levels and malignant progression of a variety of cancer types [27]. However, there is no direct evidence regarding the effect of FOXO1 on the progression of NSCLC. In this study, we demonstrated that FOXO1 inhibited the cell migration and EMT phenotype of NSCLC cells by regulating the cell surface ultrastructure.

Most recent studies have shown that inhibiting EMT decreases the metastatic ability of tumors in multiple cancers. Dong et al. confirmed that FOXO1 levels were inversely correlated with the levels of EMT inducers, and FOXO1 can bind to the ZEB2 promoter to inhibit the invasion and metastasis of hepatocellular carcinoma by reversing EMT [28]. In addition, FOXO1 interacts with the epithelial marker molecule E-cadherin, which is a core characteristic of EMT [29]. In recent years, the targeting of EMT-related pathways in human cell lines and murine models revealed the crucial importance of EMT in the progression and lethality of lung cancer. In our study, to explore the role of FOXO1 in lung cancer progression, we analyzed the cell proliferation and cell migration in NSCLCs and confirmed the function of FOXO1 in tumor cell migration. Our results showed that FOXO1 overexpression inhibited cell migration and invasion. Conversely, FOXO1 silencing significantly increased the cells' migration and invasion abilities, resulting in EMT. These results suggested that FOXO1 decreased the metastatic ability of NSCLCs by inhibiting the EMT.

Microvilli have a role in the formation of E-cadherin-based adherens junctions between epithelial cells, in addition to their role in cell migration [30,31]. Consistent with these reports, SEM analysis was used to detect the effect on cell ultrastructure of FOXO1. Remarkably, we observed that cells overexpressing FOXO1 had longer microvilli on the cell surface and showed enhanced cell adhesion. Moreover, we found that the length of the microvilli on the FOXO1-silenced cells was shorter than that of the control cells. However, we have little data on the mechanism by which FOXO1 regulates the length of the microvilli and how this enhances the adhesive ability of NSCLCs. One possibility is that the numerous microvilli induced by FOXO1 expression may be a prerequisite for the cells to form adherens junctions.

The present study provides new insights by demonstrating that the enhanced cell adhesion effect of FOXO1 is mediated by regulating the length of microvilli. Moreover, the present study extended the function via plasmid vector enhanced FOXO1 expression. Finally, FOXO1 was found to reduce cell migration, although the mechanism of this effect requires further investigation. In summary, these results shed light on FOX01's inhibition of the metastatic ability of NSCLCs by regulating the length of the microvilli.

\section{Abbreviations}

FOX01 (Forkhead box class 01); NSCLC (Non-small cell lung cancer); EMT (Epithelialmesenchymal transition); siRNA (small interfering RNA); DAPI (2-(4-amidinophenyl)-1Hindole-6-carboxamidine); SEM (scanning electron microscopy). 


\section{Cellular Physiology Cell Physiol Biochem 2018;48:138-148 and Biochemistry Published online: July $12,2018 \quad \begin{aligned} & \text { DOI: 10.1159/000491670 } 2018 \text { The Author(s). Published by S. Karger AG, Basel } \\ & \text { www.karger.com/cpb }\end{aligned}$}

Gao et al.: FOXO1 Inhibits Cell Migration by Regulating the Length of Microvilli

\section{Acknowledgements}

This work was supported by the National Natural Science Foundation of China [grant numbers 81502587, 81200570, 81703000], Haiyan Foundation of the Third Affiliated Hospital of Harbin Medical University [grant number JJQN2017-09], Innovation Foundation of Harbin Medical University [grant number 2017LCZX93], and Heilongjiang Provincial Health and Family Planning Commission [grant number 2003125].

\section{Disclosure Statement}

The authors declare that they have no conflict of interests regarding the research for this work.

\section{References}

1 Xue F, Zhu L, Meng QW, Wang L, Chen XS, Zhao YB, Xing Y, Wang XY, Cai L: FAT10 is associated with the malignancy and drug resistance of non-small-cell lung cancer. Onco Targets Ther 2016;9:4397-4409.

-2 McDoniels-Silvers AL, Nimri CF, Stoner GD, Lubet RA, You M: Differential gene expression in human lung adenocarcinomas and squamous cell carcinomas. Clin Cancer Res 2002;8:1127-1138.

3 Sakashita S, Sakashita M, Sound Tsao M: Genes and pathology of non-small cell lung carcinoma. Semin Oncol 2014;41:28-39.

4 Parsons A, Daley A, Begh R, Aveyard P: Influence of smoking cessation after diagnosis of early stage lung cancer on prognosis: systematic review of observational studies with meta-analysis. BMJ 2010;340:b5569.

5 Zhang Q, Zhang X, Yan H, Jiang B, Xu C, Yang J, Chen Z, Su J, Wu YL, Zhou Q: Effects of epidermal growth factor receptor-tyrosine kinase inhibitors alone on EGFR-mutant non-small cell lung cancer with brain metastasis. Thoracic cancer 2016;7:648-654.

6 Xi Y, Wang L, Sun C, Yang C, Zhang F, Li D: The novel miR-9501 inhibits cell proliferation, migration and activates apoptosis in non-small cell lung cancer. Med Oncol 2016;33:124.

7 Kim CG, Lee H, Gupta N, Ramachandran S, Kaushik I, Srivastava S, Kim SH, Srivastava SK: Role of Forkhead Box Class $O$ proteins in cancer progression and metastasis. Semin Cancer Biol 2017

8 Lam EW, Brosens JJ, Gomes AR, Koo CY: Forkhead box proteins: tuning forks for transcriptional harmony. Nat Rev Cancer 2013;13:482-495.

-9 Reagan-Shaw S, Ahmad N: RNA interference-mediated depletion of phosphoinositide 3-kinase activates forkhead box class $\mathrm{O}$ transcription factors and induces cell cycle arrest and apoptosis in breast carcinoma cells. Cancer Res 2006;66:1062-1069.

10 Wang Y, Zhou Y: FOXO transcription factors: their clinical significance and regulation. 2014;2014:925350.

11 Zhang X, Yalcin S, Lee DF, Yeh TY, Lee SM, Su J, Mungamuri SK, Rimmele P, Kennedy M, Sellers R, Landthaler M, Tuschl T, Chi NW, Lemischka I, Keller G, Ghaffari S: FOXO1 is an essential regulator of pluripotency in human embryonic stem cells. Nat Cell Biol 2011;13:1092-1099.

12 Kikuno N, Shiina H, Urakami S, Kawamoto K, Hirata H, Tanaka Y, Place RF, Pookot D, Majid S, Igawa M, Dahiya R: Knockdown of astrocyte-elevated gene-1 inhibits prostate cancer progression through upregulation of FOXO3a activity. Oncogene 2007;26:7647-7655.

13 Essaghir A, Dif N, Marbehant CY, Coffer PJ, Demoulin JB: The transcription of FOXO genes is stimulated by FOXO3 and repressed by growth factors. J Biol Chem 2009;284:10334-10342.

14 Shiota M, Song Y, Yokomizo A, Kiyoshima K, Tada Y, Uchino H, Uchiumi T, Inokuchi J, Oda Y, Kuroiwa K, Tatsugami K, Naito S: Foxo3a suppression of urothelial cancer invasiveness through Twist1, Y-box-binding protein 1, and E-cadherin regulation. Clin Cancer Res 2010;16:5654-5663.

15 Su B, Gao L, Baranowski C, Gillard B, Wang J, Ransom R, Ko HK, Gelman IH: A genome-wide RNAi screen identifies FOXO4 as a metastasis-suppressor through counteracting PI3K/AKT signal pathway in prostate cancer. PLoS One 2014;9:e101411. 


\section{Cellular Physiology Cell Physiol Biochem 2018;48:138-148 \begin{tabular}{l|l} 
DOI: 10.1159/000491670 & $\begin{array}{l}\text { O 2018 The Author(s). Published by S. Karger AG, Basel } \\
\text { www.karger.com/cpb }\end{array}$
\end{tabular} \\ Gao et al.: FOXO1 Inhibits Cell Migration by Regulating the Length of Microvilli}

16 Feng X, Wu Z, Wu Y, Hankey W, Prior TW, Li L, Ganju RK, Shen R, Zou X: Cdc25A regulates matrix metalloprotease 1 through Foxo1 and mediates metastasis of breast cancer cells. Mol Cell Biol 2011;31:3457-3471.

17 Zhang J, Yan Y, Yang Y, Wang L, Li M, Wang J, Liu X, Duan X, Wang J: High Infiltration of Tumor-Associated Macrophages Influences Poor Prognosis in Human Gastric Cancer Patients, Associates With the Phenomenon of EMT. Medicine (Baltimore) 2016;95:e2636.

-18 Li T, Xie J, Shen C, Cheng D, Shi Y, Wu Z, Deng X, Chen H, Shen B, Peng C, Li H, Zhan Q, Zhu Z: Upregulation of long noncoding RNA ZEB1-AS1 promotes tumor metastasis and predicts poor prognosis in hepatocellular carcinoma. Oncogene 2016;35:1575-1584.

19 Chen Y, Zhang K, Li Y, He Q: Estrogen-related receptor alpha participates transforming growth factor-beta (TGF-beta) induced epithelial-mesenchymal transition of osteosarcoma cells. Cell adhes Migr 2017;11:338346.

-20 Hirano T, Satow R, Kato A, Tamura M, Murayama Y, Saya H, Kojima H, Nagano T, Okabe T, Fukami K: Identification of novel small compounds that restore E-cadherin expression and inhibit tumor cell motility and invasiveness. Biochem Pharmacol 2013;86:1419-1429.

21 Xie R, Wang X, Qi G, Wu Z, Wei R, Li P, Zhang D: DDR1 enhances invasion and metastasis of gastric cancer via epithelial-mesenchymal transition. Tumour Biol 2016;37:12049-12059.

22 Sessa L, Gatti E, Zeni F, Antonelli A, Catucci A, Koch M, Pompilio G, Fritz G, Raucci A, Bianchi ME: The receptor for advanced glycation end-products (RAGE) is only present in mammals, and belongs to a family of cell adhesion molecules (CAMs). PLoS One 2014;9:e86903.

23 Xu ZH, Shun WW, Hang JB, Gao BL, Hu JA: Posttranslational modifications of FOXO1 regulate epidermal growth factor receptor tyrosine kinase inhibitor resistance for non-small cell lung cancer cells. Tumour Biol 2015;36:5485-5495.

-24 Guertin DA, Stevens DM, Thoreen CC, Burds AA, Kalaany NY, Moffat J, Brown M, Fitzgerald KJ, Sabatini DM: Ablation in mice of the mTORC components raptor, rictor, or mLST8 reveals that mTORC2 is required for signaling to Akt-FOXO and PKCalpha, but not S6K1 Dev Cell 2006;11:859-871.

25 Ma J, Wang N, Zhang Y, Wang C, Ge T, Jin H, Deng X, Huo X, Gu D, Ge Z, Chu W, Jiang L, Qin W: KDM6B Elicits Cell Apoptosis by Promoting Nuclear Translocation of FOXO1 in Non-Small Cell Lung Cancer. Cell Physiol Biochem 2015;37:201-213.

26 Zou J, Hong L, Luo C, Li Z, Zhu Y, Huang T, Zhang Y, Yuan H, Hu Y, Wen T, Zhuang W, Cai B, Zhang X, Huang J, Cheng J: Metformin inhibits estrogen-dependent endometrial cancer cell growth by activating the AMPKFOX01 signal pathway. Cancer Sci 2016;107:1806-1817.

27 Kan H, Huang Y, Li X, Liu D, Chen J, Shu M: Zinc finger protein ZBTB20 is an independent prognostic marker and promotes tumor growth of human hepatocellular carcinoma by repressing Fox01 Oncotarget 2016;7:14336-14349.

28 Dong T, Zhang Y, Chen Y, Liu P, An T, Zhang J, Yang H, Zhu W, Yang X: FOXO1 inhibits the invasion and metastasis of hepatocellular carcinoma by reversing ZEB2-induced epithelial-mesenchymal transition. Oncotarget 2017;8:1703-1713.

-29 Du M, Wang Q Li W, Ma X, Wu L, Guo F, Zhao S, Huang F, Wang H, Qin G: Overexpression of FOX01 ameliorates the podocyte epithelial-mesenchymal transition induced by high glucose in vitro and in vivo. Biochem Biophys Res Commun 2016;471:416-422.

30 Mattila PK, Lappalainen P: Filopodia: molecular architecture and cellular functions. Nat Rev Mol Cell Biol 2008;9:446-454.

31 Medeiros NA, Burnette DT, Forscher P: Myosin II functions in actin-bundle turnover in neuronal growth cones. Nat Cell Biol 2006;8:215-226. 